\title{
MULTICAST ROUTING WITH QUALITY OF SERVICE CONSTRAINTS IN THE AD HOC WIRELESS NETWORKS
}

\author{
Abdellah Idrissi and Fadoua Yakine \\ Department of Computer Science, Faculty of Sciences, \\ Research Computer Science Laboratory (LRI), University Mohammed V-Agdal, Rabat, Morocco
}

Received 2013-12-21; Revised 2014-04-14; Accepted 2014-04-30

\begin{abstract}
The recent multimedia applications and services are very demanding in terms of Quality of Service (QoS). This creates new challenges in ensuring QoS when delivering those services over wireless networks. Motivated by the need of supporting high quality multicast applications in wireless ad hoc networks, we propose a network topology that can minimize the power when connecting the source node to the destination nodes in multicast sessions with the respect of the QoS provisions. We formulated the problem as integer linear programming problem with a set of energy and QoS constraints. We minimize the total power of energy used by nodes while satisfying QoS constraints (Bandwidth and maximum delay) that are crucial to wireless ad hoc network performance.
\end{abstract}

Keywords: Ad Hoc Network, Wireless, Routing, Multicast, Energy-Efficient Routing, QoS, Topology Control

\section{INTRODUCTION}

A wireless ad hoc network is a type of wireless networks where communication between nodes does not require any established infrastructure or centralized administration. The wireless ad hoc nodes are communication end-points as well as routers. To establish the communication between two non direct neighbors end-nodes, the relay of messages by intermediate nodes is needed.

A wireless ad hoc network is a type of wireless networks where communication between nodes does not require any established infrastructure or centralized administration. The wireless ad hoc nodes are communication end-points as well as routers. To establish the communication between two non direct neighbors end-nodes, the relay of messages by intermediate nodes is needed.

Most of the existing works about the subject deal with QoS routing or admission control (Chen and Nahrstedt, 1999). The QoS requirements are most often expressed in terms of delay and bandwidth. In multi-hop ad hoc networks, those parameters are particularly dependent on the network topology. It has been proven that the QoS routing with multiple constraints is NP Complete problem (Thenmozhi and Lakshmipathi, 2010). Hence, there are no exact studies in literature for providing algorithms that can overall satisfy QoS requirements. Some Nodes of the network could be overloaded making impossible to construct QoS routes (Li et al., 2001). So, designing a routing algorithm supporting QoS is essential.

The topology of an ad hoc network depends on controllable factors such as the transmitting power and antenna directions of the nodes. The topology control in ad hoc networks is essential because a wrong topology could considerably reduce the capacity, increase the end to-end packet delay and decrease the robustness to node failures. If the topology is too sparse, there would be less choices for routing (hence, some nodes could be overloaded) and the average endto-end hop-count will be high. On the other hand, if the topology is too dense, there would be more routing choices, but the power consumption of the system would be highly increased (Pan and Xiao, 2006).

Corresponding Author: Abdellah Idrissi, Department of Computer Science, Faculty of Science, Computer Science Laboratory (LRI), University Mohammed V-Agdal, Rabat, Morocco 
The concept of multicast is a very useful mechanism that supports communication from one source node to many destinations. The multicast routing deals with the construction of a multicast tree, which is originated at a source node and covers all destination nodes. The QoS multicast tries to find the multicast tree while every internal path going from the source to the destination conforms to the QoS requirements (Wu and Jia, 2007).

We are mainly interested in the formulations related to the minimum power broadcast trees for Wireless Networks (Das et al., 2003). We propose to extend them to support QoS requirements. This paper discusses route optimization of multicast session with QoS constraints in wireless ad hoc networks. Our objective is to reduce the total power of energy used by nodes while satisfying required QoS parameters. Our QoS criteria are delay and traffic demands.

\section{MATERIALS AND METHODS}

There are several researches that have already discussed topology control in wireless ad hoc networks. Most of the works referring to the construction and maintenance of a network topology with required connectivity used minimal power consumption. Lloyd et al. (2005) addressed the topology control problem with several optimization objectives including both minimizing the maximum energy consumption used by any node and minimizing the total energy consumption used by all nodes. For static networks, two centralized algorithms were proposed for constructing connected and biconnected networks with the minimization of the maximal transmitting power for each node. Two additional distributed control heuristics were further proposed, such as Local Information No Topology (LINT) and Local Information Link state Topology (LILT), to deal with the topological changes (Ramanathan and Rosales-Hain, 2000). Li et al. (2003) focalized on the network connectivity with low power consumption and proposed a minimum spanning tree based topology control algorithm. In CBTC (Li et al., 2001), each node grows its transmitting power until it finds at least one neighbor in every cone of degree $\alpha$. The basic idea of the algorithm is that a node $u$ tries to find the minimum power $\mathrm{P}_{\mathrm{u}}, \alpha$ such that it ensures some node is present in every cone of angel $\alpha$ around u. Huang et al. (2002) have extended this idea to the case of directional antennas. Marsan et al. (2002) addressed the problem of determining an optimal Bluetooth topology, which minimizes the maximum traffic load of nodes. Jia et al. (2004) discussed the construction of network topology that meets the QoS requirements in terms of end-to-end delay and bandwidth and minimizes the maximum power consumption per node.

A lot of works have been proposed for the problems of minimizing the energy consumption for broadcasting and multicasting in wireless ad hoc networks, addressed as the Minimum-Energy Broadcast (MEB) problem and Minimum-Energy Multicast (MEM) problem respectively. The MEM problem was proved to be NP hard (Guo and Yang, 2004).

Some previous works on the issue of energy efficiency had made great progress. Singh et al. (1998) proposed five power-aware metrics that can be easily incorporated into existing routing protocols. These metrics are based on battery power consumption at nodes. Kawadia and Kumar (2003) proposed to have clusters with different level of transmitting powers, where each may belong to more than one cluster of different power levels and routing could be determined using the least power paths. Wieselthier et al. (2000), studied the problem of reducing the total energy cost of a broadcast/multicast tree by adjusting the energy power of each node. They proposed three greedy heuristics namely Broadcasting Incremental Power (BIP), Minimum Spanning Tree (MST) and the Shortest-Path Tree (SPT). Wan et al. (2001) proposed a quantitative analysis to measure the performance of these heuristics. The MEM problem was studied in the same way as the MEB problem. The resulting minimum-energy broadcast tree is pruned to construct the minimum-energy multicast tree by eliminating from it all transmissions not needed to reach the member of the multicast set. When applied to BIP, the resulting algorithm is called Multicast Incremental Power (MIP) (Wieselthier et al., 2000).

Since the MEM problem is known to be NP-hard, many mathematical programming approaches have been introduced in several works. Das et al. (2003) presented three linear integer programming models for MEM, without any experimental implementation. Bauer et al. (2008) presented a model for numerically evaluating the BIP heuristic in large networks based on multicommodity flow and a Lagrangean relaxation. Sabari and Duraiswamy (2009) proposed an Ant based algorithm for Multicast routing in mobile ad hoc networks. Maruthamuthu and Sankaralingam (2011) presented Energy aware Multiple constraints QoS Routing Protocol with Dynamic Mobility prediction (EMQRPDM), which is a source based reactive protocol for MANET. In objective to minimize the consumption of batteries power, (Idrissi, 2012) presented a method based on an 
adaptation of the Dijkstra's algorithm to the MANET problem called MANED and proposed in (Idrissi et al., 2006) an approach based on an adaptation of the A star algorithm to minimize the consuption of energy.

We think that integer programming formulations can be useful for obtaining exact or approximate solutions to MEM problems. We present hereafter the system modeling.

We think that integer programming formulations can be useful for obtaining exact or approximate solutions to MEM problems. We present hereafter the system modeling.

\section{SYSTEM MODELING}

In this study, we will use a group of notations. We assume a fixed $\mathrm{N}$-node network with their locations. The network is described by a graph $\mathrm{G}=(\mathrm{V}, \mathrm{E})$, where $\mathrm{V}$ is the group of $\mathrm{N}$ nodes and $\mathrm{E}$ is a set of edges. $\mathrm{M}$ denotes the multicast set, where MİV $\backslash\{s\}$. Any node can be used as an intermediate node to reach other nodes in the network. We used the widely adopted transmitting power model for radio networks:

$$
P_{i j}=d_{i j}^{\alpha}
$$

Where:

$\mathrm{P}_{\mathrm{ij}} \quad=$ The transmitting power used by the network node $\mathrm{i}$ to reach $\mathrm{j}$,

$\mathrm{d}_{\mathrm{ij}} \quad=$ The distance between nodes

$\mathrm{i}$ and $\mathrm{j}$ and $\alpha=\mathrm{a}$ parameter corresponding to the characteristics of the communication medium (it ranges between 2 and 4)

Let $\mathrm{P}_{\mathrm{i}}$ denotes the transmitting power of node $\mathrm{i}$ where $1 \leq \mathrm{i} \leq \mathrm{N}$. We assume that each node can adjust its power level depending on the transmission power of nodes and their distances. The connections between nodes depend on their transmission power. An edge (i, $\mathrm{j}) \in \mathrm{E}$ if $\mathrm{P}_{\mathrm{i}} \geq\left(\mathrm{d}_{\mathrm{ij}}\right)^{\alpha}$.

The total energy cost $P_{\text {total }}$ is total energy consumed by each node in the tree. It can be represented as Equation 1:

$$
P_{\text {total }}=\sum_{i=1}^{N} P_{i}
$$

Let $\Delta$ and $\lambda_{\mathrm{s}, \mathrm{d}}$ denote the maximally permitted hopcount and the traffic demand from a node source $s$ to a destination node $\mathrm{d}$ (where $\mathrm{d} \in \mathrm{M}$ ) for the multicast request.
In a multicast scenario, a source node $s \in \mathrm{V}$ sends a message to a set $M . M=\left\{d_{1}, d_{2}, \ldots, d_{m}\right\}$ denotes the multicast group members. Our topology control problem can be formally defined as: Given a multicast request $(\mathrm{s}, \mathrm{M})$ and $\mathrm{p}_{\mathrm{i}}$ for each node $\mathrm{i}, 1 \leq \mathrm{i} \leq \mathrm{N}$, our objective is to minimize $\mathrm{P}_{\text {total }}$ when constructing a multicast tree rooted from $s$ and spanning all nodes in $M$ such that all the traffic demands can be transmitted with the respect of the permitted hop-count.

\section{PROBLEM FORMULATION}

For the purpose of formulating the problem of QoS multicast routing, we define the following variables:

- $\mathrm{X}_{\mathrm{ij}}$ is a Boolean decision variable which is equal to one if there is a link between node $\mathrm{i}$ and node $\mathrm{j}$ and zero otherwise

- $\mathrm{X}_{\mathrm{ij}}^{\mathrm{sd}}$ is a Boolean decision variable that we have introduced in the model to ensure flow constraint. $X_{i j}^{s d}=1$ if the path linking $s$ to $d$ goes through link $(i, j)$, else $X_{i j}^{s d}=0$; Where $d$ is a destination node from the set $M$

- $\mathrm{P}_{\mathrm{i}}$ is the transmission power of node $\mathrm{i}$.

Our objective is to minimize the overall transmitting energy of all nodes in the multicast session

\subsection{Objective Function:}

$$
\mathrm{P}_{\text {total }}=\min \sum_{\mathrm{i}=1}^{\mathrm{N}} \mathrm{P}_{\mathrm{i}}
$$

\subsection{Multicast Routing Constraints}

\subsubsection{Power Constraints}

The constraint (3) defines the relations between the transmitting power $\mathrm{p}_{\mathrm{i}}$ and the binary variables $\mathrm{X}_{\mathrm{ij}}$ :

$$
P_{i}-P_{i j} X_{i j} \geq 0 ; \forall(i, j) \in v, i \neq j
$$

The power matrix of a network, $\mathrm{P}$, is defined to be an $\mathrm{N}^{\prime} \mathrm{N}$ matrix whose $(\mathrm{i}, \mathrm{j})^{\text {th }}$ element, $\mathrm{P}_{\mathrm{ij}}$, is the power needed by node $\mathrm{i}$ to reach node $\mathrm{j} . \mathrm{P}_{\mathrm{ij}}$ is described by (4):

$$
P_{i j}=\left[\left(x_{i}-x_{j}\right)^{2}+\left(y_{i}-y_{j}\right)^{2}\right]^{\alpha / 2}=d_{i j}^{\alpha} ; 1 \leq i, j \leq N
$$

where, $\left(\mathrm{x}_{\mathrm{i}}, \mathrm{y}_{\mathrm{i}}\right)$ are the coordinates of the network nodes and $d_{i j}$ is the Euclidean distance separating $i$ from $j$. 


\subsection{Topology Constraints}

The constraint (5) defines the condition that the source node of the multicast session should transmit at least once:

$$
\sum_{j=1}^{N} X_{i j} \geq 1 ; i=s, i \neq j
$$

The node reachability constraints (6) expresse that there is no condition about the number of transmissions done by node $\mathrm{i}$ :

$$
\sum_{j=1}^{N} X_{i j}=1 ; \forall j \in M, i \neq j
$$

In order to avoid any loops in the final tree, constraints (7) are added. They specify that a node can transmit only if it receives a transmission from another node (other than the source s):

$$
\sum_{j=1}^{N} X_{i j} \leq(N-1) \sum_{j=1}^{N} X_{j i} ; \forall(i, j) \in\{v-s\}, i \neq j
$$

\subsection{QoS Constraints}

In this study, two QoS parameters are considered:

\subsection{Delay Constraint}

The constraint (8) warrants that the hop-count $\Delta$ for the multicast tree does not surpass the pre-specified bound. To provide delay constrained session, this parameter should be guaranteed:

$$
\sum(\mathrm{i}, \mathrm{j}) \mathrm{x}_{\mathrm{i}, \mathrm{j}}^{\mathrm{s}, \mathrm{d}} \leq \Delta, \forall \mathrm{s} \in \mathrm{V}, \forall \mathrm{d} \in \mathrm{M}
$$

\subsection{Bandwidth Constraint}

Constraint (9) is added to guarantee bandwidth requirements. It ensures that the bandwidth capacity of a node is within the total transmission and reception at the same node:

$$
\sum(\mathrm{s}, \mathrm{d}) \sum_{\mathrm{i}} \mathrm{x}_{\mathrm{i}, \mathrm{j}}^{\mathrm{s}, \mathrm{d}} \lambda_{\mathrm{s}, \mathrm{d}}+\sum_{(\mathrm{s}, \mathrm{d})} \sum_{\mathrm{j}} \mathrm{x}_{\mathrm{i}, \mathrm{j}}^{\mathrm{s}, \mathrm{d}} \lambda_{\mathrm{s}, \mathrm{d}} \leq \mathrm{B}, \forall \mathrm{d} \in
$$

\subsection{Route (Flow) Constraints}

Constraint (10) is for flow conservation, $\mathrm{X}_{\mathrm{ij}}^{\mathrm{sd}}=1$ if the path linking $s$ to $d$ goes through link $(i, j)$, else $X_{i j}^{s d}=0$. This constraint states that the entire traffics from $s$ to $d$ and passing through any relay node must be equal to the traffic entering this same node:

$$
\sum_{j} X_{i j}^{s, d}-\sum_{j} X_{i j}^{s, d}=\left\{\begin{array}{l}
1 \text { if } s=1 \\
-1 \text { if } d=i \forall i \in v \\
0 \text { otherwise }
\end{array}\right.
$$

\subsection{Route Validity}

Constraint (11) ensures that the route between each node-pair is valid. It states that traffic is circulating from node $\mathrm{i}$ to node $\mathrm{j}$ only when the link $(\mathrm{i}, \mathrm{j})$ exists:

$\sum(\mathrm{i}, \mathrm{j}) \mathrm{x}_{\mathrm{i}, \mathrm{j}}^{\mathrm{s}, \mathrm{d}} \leq \mathrm{x}_{\mathrm{tj}} \forall(\mathrm{s}, \mathrm{M})$

\subsection{Other Constraints}

$$
\begin{aligned}
& \mathrm{x}_{\mathrm{i}, \mathrm{j}}^{\mathrm{s}, \mathrm{d}}=1, \text { or } 0, \mathrm{x}_{\mathrm{i}, \mathrm{j}}=1 \operatorname{or} 0 \forall(\mathrm{i}, \mathrm{j}) \in \mathrm{V},(\mathrm{s}, \mathrm{M}) \\
& \mathrm{P}_{\mathrm{i}} \geq 0, \forall(\mathrm{i}, \mathrm{j}) \in \mathrm{v},(\mathrm{s}, \mathrm{M})
\end{aligned}
$$

The proposed algorithm can be performed as the following steps:

Generate $\mathrm{V}$ a N-Node set with their locations. Compute the P matrix $(\mathrm{N} \times \mathrm{N})$ of the network using (4). Input: Network is a graph of $\mathrm{N}$ nodes, $\mathrm{P}$ matrix, Multicast request set: $\mathrm{R}=\left\{\left(\mathrm{s}, \mathrm{M}, \lambda_{\mathrm{s}, \mathrm{d}}, \Delta\right)\right\}$ and $\mathrm{d} \in \mathrm{M}$. $\mathrm{s}$ is the source Node, $\mathrm{M}$ the destination nodes, $\lambda_{\mathrm{s}, \mathrm{d}}$ is the traffic demand for node pair source (s) to destination (d) and $\Delta$ denotes the maximally allowed hop-count for the multicast tree.

Output: Multicast tree originating from s and spanning the destination nodes $\mathrm{M}, \mathrm{P}_{\text {total }}$ of the tree.

1. Run the ILP model with previous Equation 2-13 to find the minimum energy tree from $s$ to each terminal $\mathrm{d} \in \mathrm{M}$ with the respect of the hop-count bound $\Delta$ and the traffic demands $\lambda_{\mathrm{s}, \mathrm{d}}$.

2. Return the optimal solution of the ILP.

3. END

\section{SIMULATION RESULTS}

To compute the solution for this ILP model, many tools can be employed like Matlab, Lpsolve, CPLEX, etc. To simulate this model respecting the previous Equation 2-13, we used CPLEX (IBM ILOG CPLEX V12.1, 2009). CPLEX is an optimization software developed by ILOG for solving linear, integer and quadratic programming problems.

All the computational experiments are performed on a personal computer with $4 \mathrm{~GB}$ RAM and $2.4 \mathrm{GHz}$, Core 2 Duo Intel processor. The Simulations were carried out on a $30 \times 30$ meter two dimensional free space region. 
We assume that the number of nodes is $15(\mathrm{~N}=15)$. The node coordinates are picked randomly (Fig. 1). All network nodes have the same bandwidth $(\mathrm{B}=11 \mathrm{Mbps})$. Every multicast request is a set $\mathrm{R}=\left\{\left(\mathrm{s}, \mathrm{M}, \lambda_{\mathrm{s}, \mathrm{d}}, \Delta\right)\right\}$. To generate the set $\mathrm{R}$, we developed a script that choose randomly a node from the $\mathrm{N}$ nodes to be the source $\mathrm{s}$ of the request then we choose a random set $\mathrm{M}$ of destination nodes from the remaining nodes $\{\mathrm{N}-\mathrm{s}\}$. To assign traffic demands $\lambda_{\mathrm{s}, \mathrm{d}}$ for each pair $(\mathrm{s}, \mathrm{d})$ with $\mathrm{d} \in \mathrm{M}$, we used a random function of a normal distribution with variance equal to $0.5 \mu_{\mathrm{m}}$ ( $\mu_{\mathrm{m}}$ is the mean value of the normal distribution function). The average bandwidth requirement per request is $\mu_{\mathrm{m}}$. The maximum value which we set for the Multicast tree hop-count is $\Delta_{\max }=2 \mathrm{~N} / 3$.

We initiate 8 requests of multicast sessions. The source (2nd column), destinations M (3rd column) and traffic demand for each (s,d) with $d \in M$ (4th column) of those requests are generated as we mentioned above. The average traffic amount per request is $\lambda_{\mathrm{m}}=0.01 \mathrm{~B}$. The routing information concerning each request of our first experiment are showed in Table 1.

In each run of the simulation, we execute the algorithm in the network and report the route, the delay and the total energy needed for constructing a multicast tree originated at $\mathrm{s}$ and addressing all the destinations nodes $\mathrm{M}$. The delay of the multicast tree is defined as the maximum value of delay in the route linking the source $\mathrm{s}$ to every destination node $\mathrm{d}$ from M (Chen and Sun, 2005).

The total energy cost of the algorithm increases as $M$ increases. The reason is when the number of destinations increases, the number of traffic demands to satisfy increases too causing much more energy cost.

In the second experiment, we specify the maximum hop count permitted for the same sets of source and destinations on the same 15 nodes sample graph. The Table 2 summarises the results obtained.

Table 1. The routes per request for $\mu_{\mathrm{m}}=0.2 \mathrm{~B}$

\begin{tabular}{|c|c|c|c|c|c|c|}
\hline $\operatorname{Req}^{\circ}$ & $S$ & $\mathrm{M}$ & $\lambda_{\mathrm{s}, \mathrm{d}}(\mathrm{kbps})$ & Route & Delay of the tree & $\mathrm{P}_{\text {total }}$ \\
\hline 1 & 1 & $\{9\}$ & 1098.8 & $1 \rightarrow 10 \rightarrow 4 \rightarrow 9$ & 3 & $97,53,37$ \\
\hline 2 & 1 & $\{6 ; 14 ; 15\}$ & $1098.2,1100.51097 .0$ & $1 \rightarrow 12 \rightarrow 14, \rightarrow 6\llcorner 13 \rightarrow 8 \rightarrow 15$ & 3 & $238.0,311$ \\
\hline 3 & 10 & $\{1 ; 2 ; 3 ; 7 ; 9 ; 14\}$ & $\begin{array}{l}1100.6,1095.9,1101.1,1100.7 \\
1101.5,1098.6\end{array}$ & や3, 10 $\rightarrow 1 \rightarrow 12 \rightarrow 14, \bigsqcup 2 \rightarrow 7\llcorner 4 \rightarrow 9$ & 3 & $427.0,332$ \\
\hline 4 & 10 & $\begin{array}{l}\{1 ; 2 ; 4 ; 5 ; 7 ; \\
8 ; 11 ; 13 ; 15\}\end{array}$ & $\begin{array}{l}1101.1,1098.6, \\
1101.7,1095.5,1102.2, \\
1100.0,1096.8,1097.51100 .4\end{array}$ & $\begin{array}{l}\gg 1 \rightarrow 13 \rightarrow 8, \rightarrow 1 \rightarrow 11 \\
10 \rightarrow 2 \rightarrow 7 \rightarrow 5, \text { ᄂ4 }\end{array}$ & 5 & $370.9,819$ \\
\hline 5 & 10 & $\begin{array}{l}\{1 ; 2 ; 3 ; 4 ; 9 \\
11 ; 12 ; 13\}\end{array}$ & $\begin{array}{l}1100.4,1098.0 \\
1099.1,1102.8 \\
1099.1,1100.1 \\
1101.6,1101.8\end{array}$ & $\begin{array}{l}\text { や13 户 } 1 \rightarrow 12 \rightarrow 3 \\
10 \rightarrow 2 \rightarrow 11,\llcorner 4 \rightarrow 9\end{array}$ & 3 & $204.0,96$ \\
\hline 6 & 12 & $\begin{array}{l}\{1 ; 2 ; 3 ; 4 ; 6 \\
10 ; 13 ; 14\}\end{array}$ & $\begin{array}{l}1100.3,1096.8 \\
1096.7,1100.9 \\
1098.5,1101.1 \\
1097.2,1100.9\end{array}$ & $\begin{array}{l}\longmapsto 13,12 \rightarrow 1 \rightarrow 10 \rightarrow 2, \\
\llcorner 3\llcorner 4,\llcorner 14 \rightarrow 6\end{array}$ & 3 & $328.1,068$ \\
\hline 7 & 4 & $\{2 ; 5 ; 7 ; 9 ; 10 ; 14\}$ & $\begin{array}{l}1101.3,1100.8 \\
1098.0,1099.3 \\
1100.9,1096.8\end{array}$ & $\begin{array}{l}4 \rightarrow 9,\llcorner 10 \rightarrow 1 \rightarrow 12, \rightarrow 14, \\
\llcorner 2 \rightarrow 7 \rightarrow 5\end{array}$ & 4 & $511.6,554$ \\
\hline 8 & 13 & $\{9 ; 10 ; 11 ; 14\}$ & $\begin{array}{l}1098.6,1097.8 \\
1102.0,1103.5\end{array}$ & 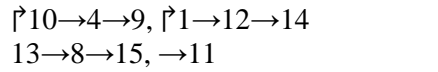 & 4 & $333.6,209$ \\
\hline
\end{tabular}

Table 2. The routes per request with max-hop count and $\mu \mathrm{m}=0.2 \mathrm{~B}$

\begin{tabular}{|c|c|c|c|c|c|}
\hline $\operatorname{Req}^{\circ}$ & $S$ & $\mathrm{M}$ & $\begin{array}{l}\text { Max } \\
\text { hopcount }\end{array}$ & Route & $\mathrm{P}_{\text {total }}$ \\
\hline 1 & 1 & $\{9\}$ & 5 & $1 \rightarrow 10 \rightarrow 4 \rightarrow 9$ & 97,5337 \\
\hline 2 & 1 & $\{6 ; 14 ; 15\}$ & 2 & $1 \rightarrow 8 \rightarrow 15,\llcorner$ 14 $\rightarrow 6$ & 384.5776 \\
\hline 3 & 10 & $\{1 ; 2 ; 3 ; 7 ; 9 ; 14\}$ & 2 & 1 † $3,10 \rightarrow 12 \rightarrow 14,\llcorner 2 \rightarrow 7,\llcorner 4 \rightarrow 9$ & 477.9231 \\
\hline 4 & 10 & $\{1 ; 2 ; 4 ; 5 ; 7 ;$ & 3 & や13, Ґ $1 \rightarrow 8 \rightarrow 15,10 \rightarrow 2 \rightarrow 7 \rightarrow 5$, Ц4 ᄂ 11 & 374.8410 \\
\hline 5 & 10 & $\{1 ; 2 ; 3 ; 4 ; 9 ; 11 ; 12 ; 13\}$ & 2 & $134 队 3$, $1 \rightarrow 12,10 \rightarrow 2 \rightarrow 11,\llcorner 4 \rightarrow 9$ & 256.8860 \\
\hline 6 & 12 & $\{1 ; 2 ; 3 ; 4 ; 6 ; 10 ; 13 ; 14\}$ & 1 & や6, 4乌阝3, $2 \leftarrow 12 \rightarrow 1,10$ اله13, ال14 & 857.8586 \\
\hline 7 & 4 & $\{2 ; 5 ; 7 ; 9 ; 10 ; 14\}$ & 2 & $\gg 5 \rightarrow 7,4 \rightarrow 6 \rightarrow 14,\llcorner 9,\llcorner 10 \rightarrow 2$ & 571.6614 \\
\hline 8 & 13 & $\{9 ; 10 ; 11 ; 14\}$ & 3 & $\gg 10 \rightarrow 4 \rightarrow 9, \gg 12 \rightarrow 14,13 \rightarrow 8 \rightarrow 15, \rightarrow 11$ & 353.3703 \\
\hline
\end{tabular}




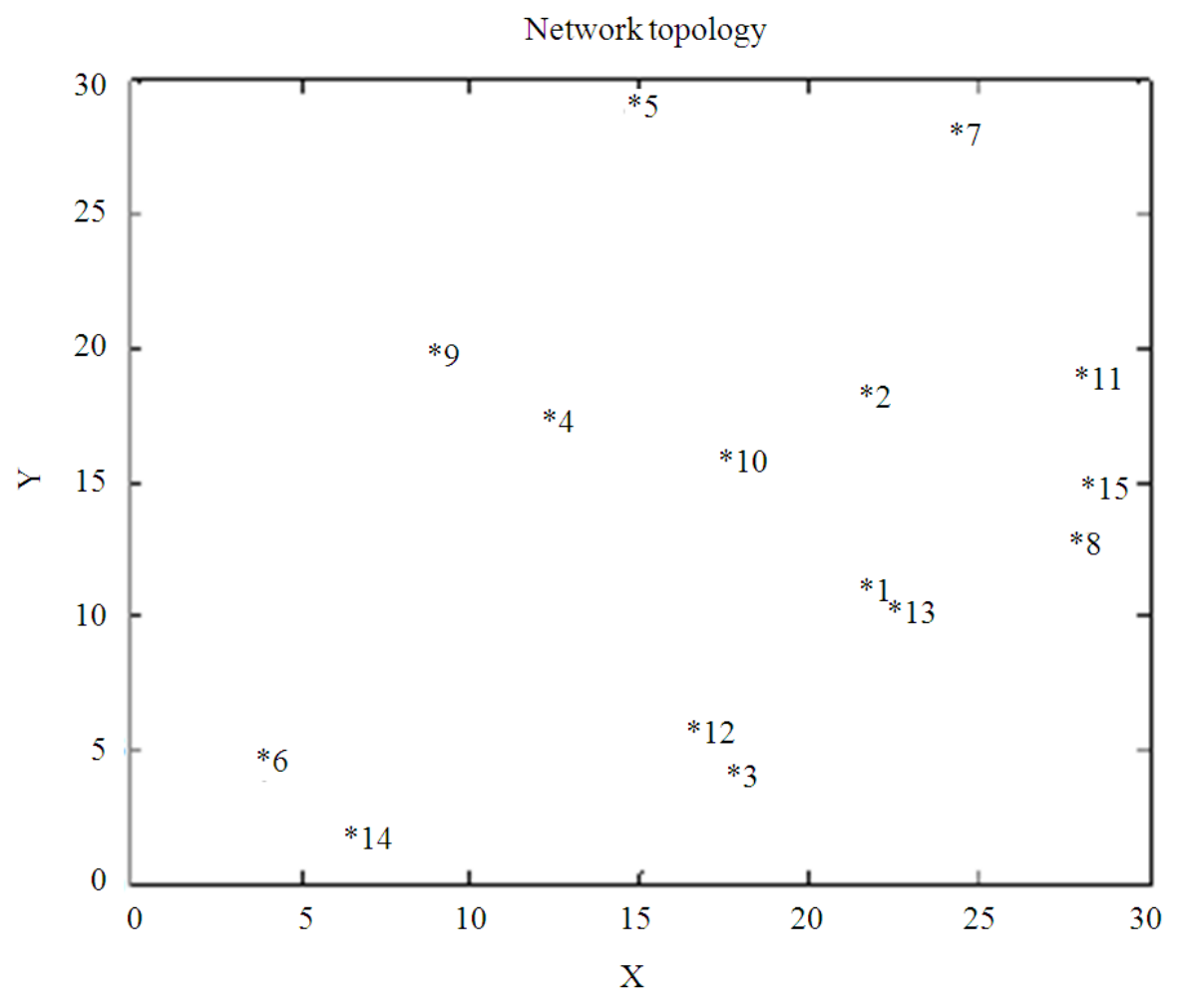

Fig. 1. The input topology number of nodes $n=15$ in $30 \times 30$ Meter

Table 2 reports the routes and the $P_{\text {total }}$ when we specify the permitted delay constraint of the multicast tree. In comparison with Table 1, we can notice the increase of the total power and the change of routes between $\mathrm{s}$ and the set of destination nodes as we reduce the hop count. The relative position of source and destinations influences the total cost of the multicast tree. The reduction of $\Delta$ to ensure delay constraint causes the increase of the cost of the multicast tree.

In the third experiment, we aim to analyze the generated topologies as the changes of $\mu_{\mathrm{m}}$. We can note that the higher values of the mean traffic demand $\left(\mu_{\mathrm{m}}\right)$ gets, the more power is required. Hence, the total power of the tree is increased. This can be noticed in Fig. 2-6. When the requested $\mu_{m}$ is small compared to $B$, topological changes occur rarely (Fig. 2-4). The edges in the topology changed, when $\mathrm{mm}$ reaches a certain threshold $\left(\mu_{m}=0.26\right.$ B) (Fig. 5). When $\mu_{m}$ is greater than $0.4 \times \mathrm{B}$, we notice that there is no topology that can satisfy the requested traffics.

As observed in these figures, when we enlarge $\mathrm{mm}$ the energy cost of the multicast tree increases because the higher $\mu_{m}$ is, the traffic demands get higher too causing the change of the topology constructed to satisfy those traffic demands and thus increasing the total energy cost of the multicast session.

In the fourth experiment, we simulated the algorithm in, a larger network, 100 node based network $(\mathrm{N}=100)$ in a $200 \times 200 \mathrm{~m}$ space region. We run the algorithm for random sources and random multicast groups. The average traffic amount per request is $\lambda_{\mathrm{m}}=0.01 \mathrm{~B}$. We have considered scenarii with a specified multicast group size $\mathrm{M}(\mathrm{M}=10,50$, 60,80 and 90). For each random $M$ set, we specified the values of $P_{\text {total }}$ needed. The results shown in Fig. 7 are the average values of 10 runs in the network.

$P_{\text {total }}$ increases slowly when $\mathrm{M}$ is small as shown in Fig. 7. $\mathrm{P}_{\text {total }}$ increases quickly when $\mathrm{M}$ is greater than $\mathrm{M}$ $=80$. We can notice that when the size of the multicast set increases, the amount of total energy gets higher which is required to obtain a feasible multicast tree.

When the $M$ size is less than $M=60$, we manage to have solution in reasonable time (the computation time is small). When $\mathrm{M}$ continues increasing, it takes a much more time to compute feasible solutions. But when M reaches 80 , we start to have no feasible solution cases. 
Abdellah Idrissi and Fadoua Yakine / Journal of Computer Science 10 (9): 1839-1849, 2014

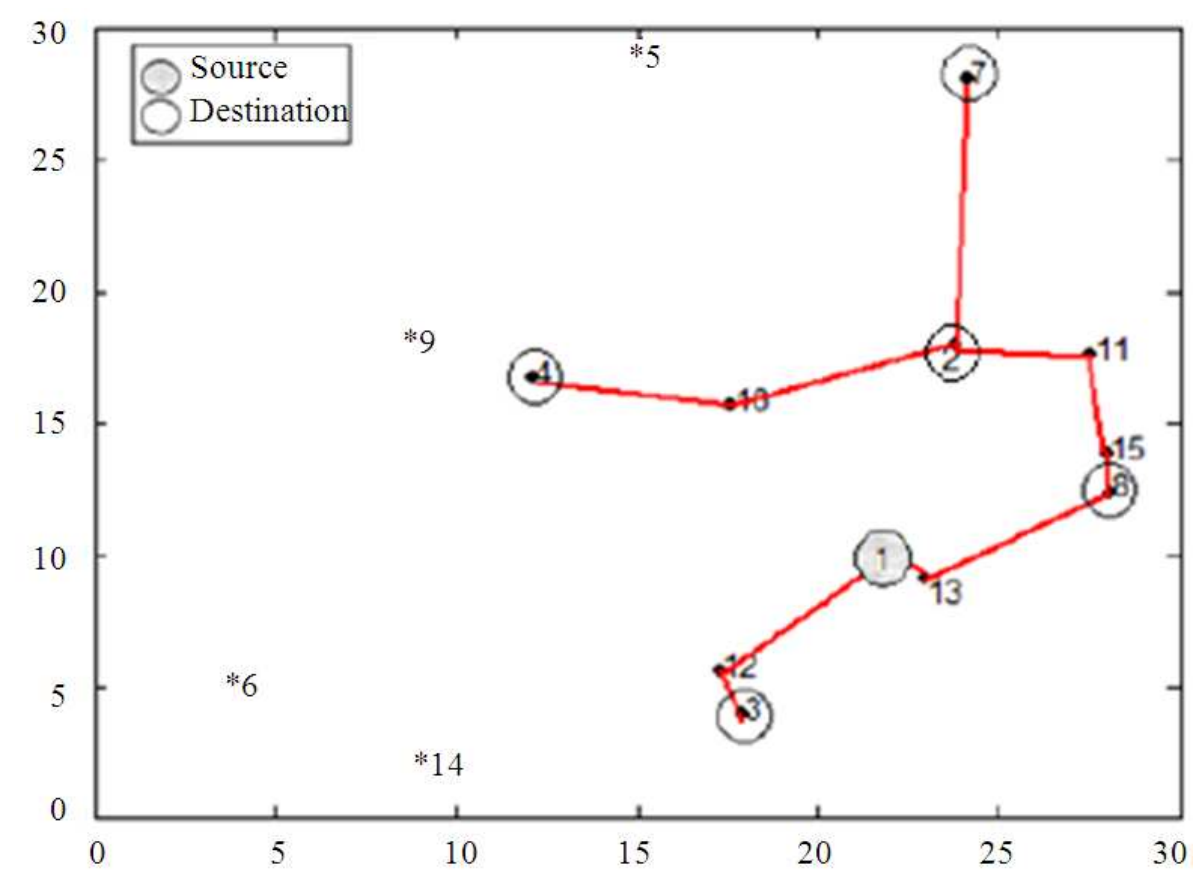

Fig. 2. The multicast tree for destinations $\{2,3,4,7,8\}$ with $\mathrm{mm} \mu_{\mathrm{m}}=0.04 \mathrm{~B}, \mathrm{P}_{\text {total }}=292.5959$

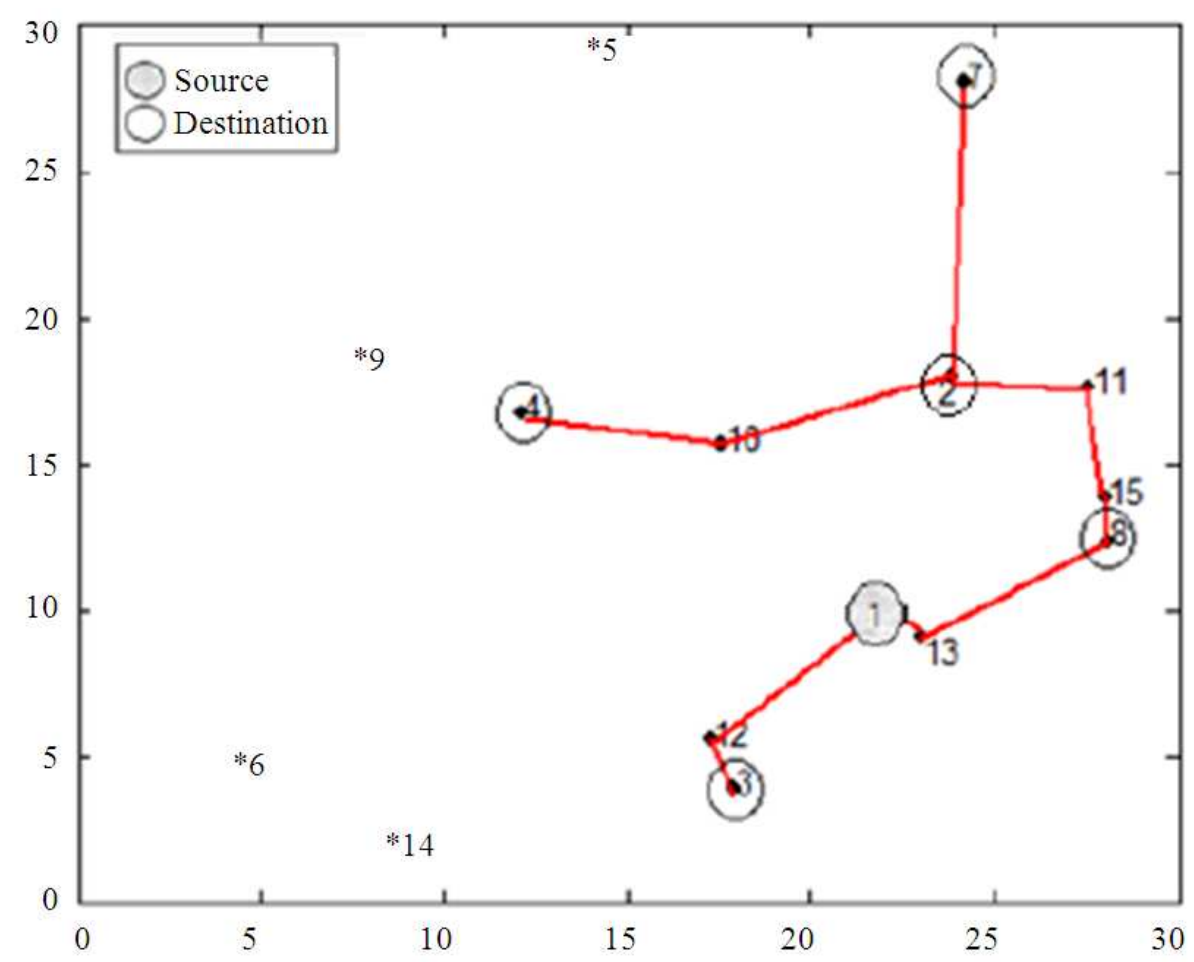

Fig. 3. The multicast tree for destinations $\{2,3,4,7,8\}$ with $\mu_{m}=0.2 B, P_{\text {total }}=292.5959$ 
Abdellah Idrissi and Fadoua Yakine / Journal of Computer Science 10 (9): 1839-1849, 2014

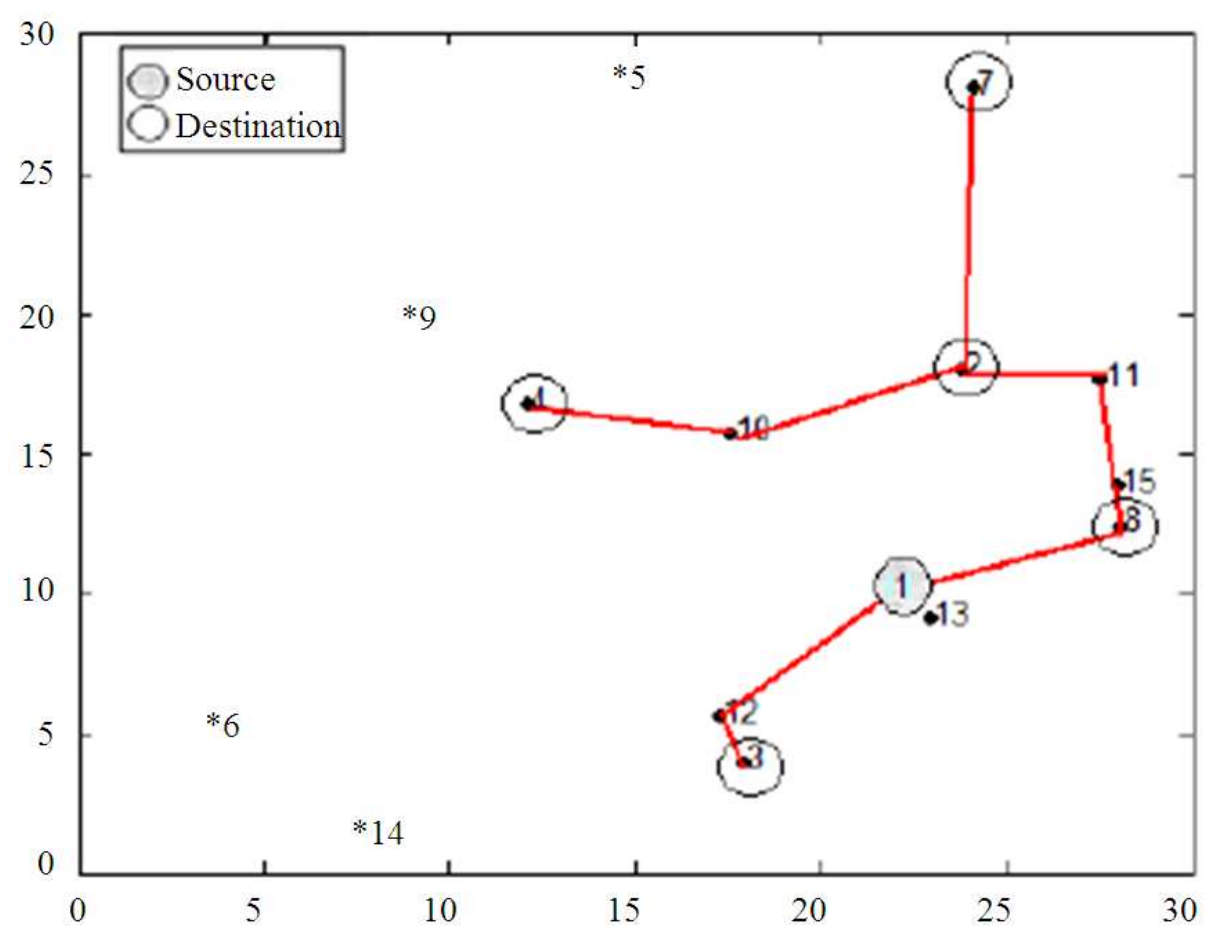

Fig. 4. The multicast tree for destinations $\{2,3,4,7,8\}$ with $\mu_{\mathrm{m}}=0.26 \mathrm{~B} \mathrm{P}_{\text {total }}=294.5638$

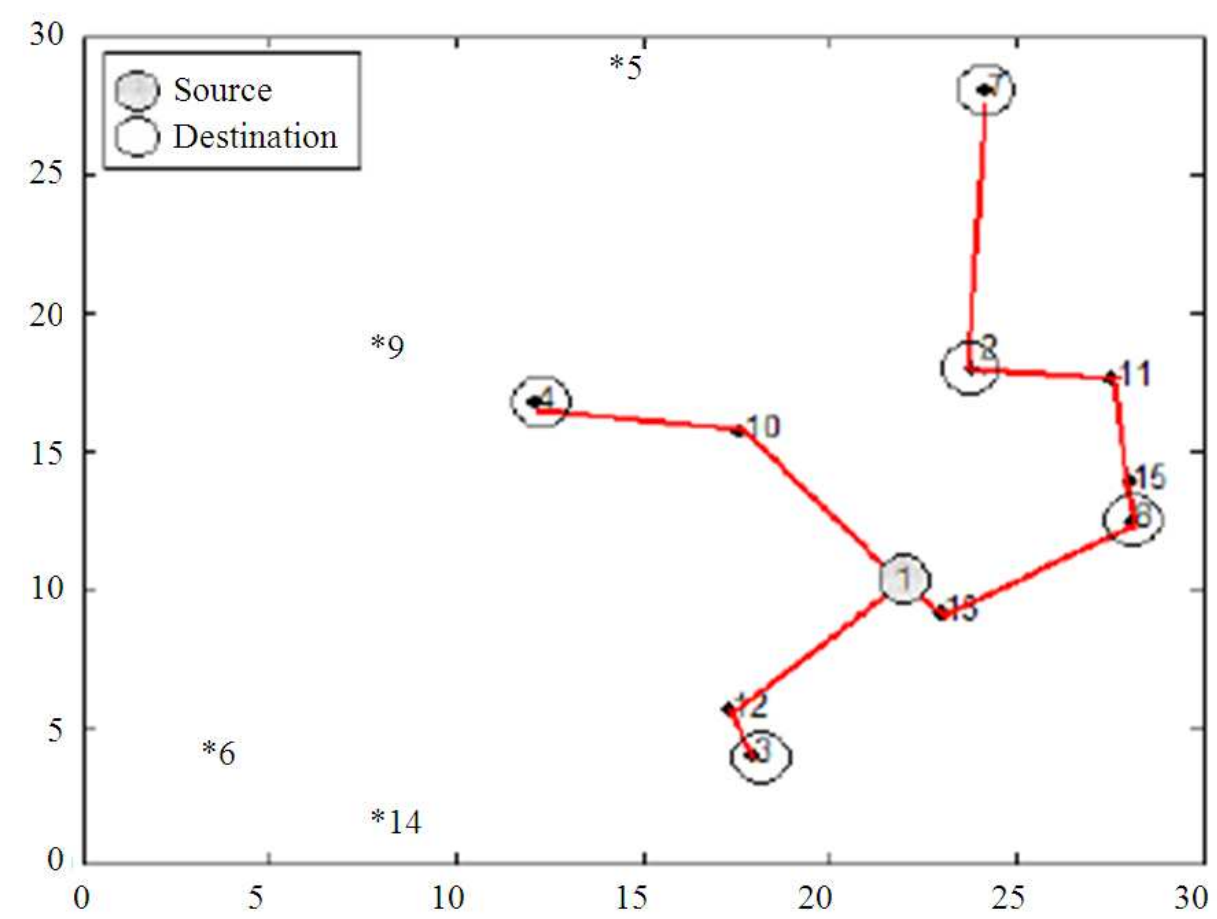

Fig. 5. The multicast tree for destinations $\{2,3,4,7,8\}$ with $\mu_{m}=0.29 B, P_{\text {total }}=300,5340$; 
Abdellah Idrissi and Fadoua Yakine / Journal of Computer Science 10 (9): 1839-1849, 2014

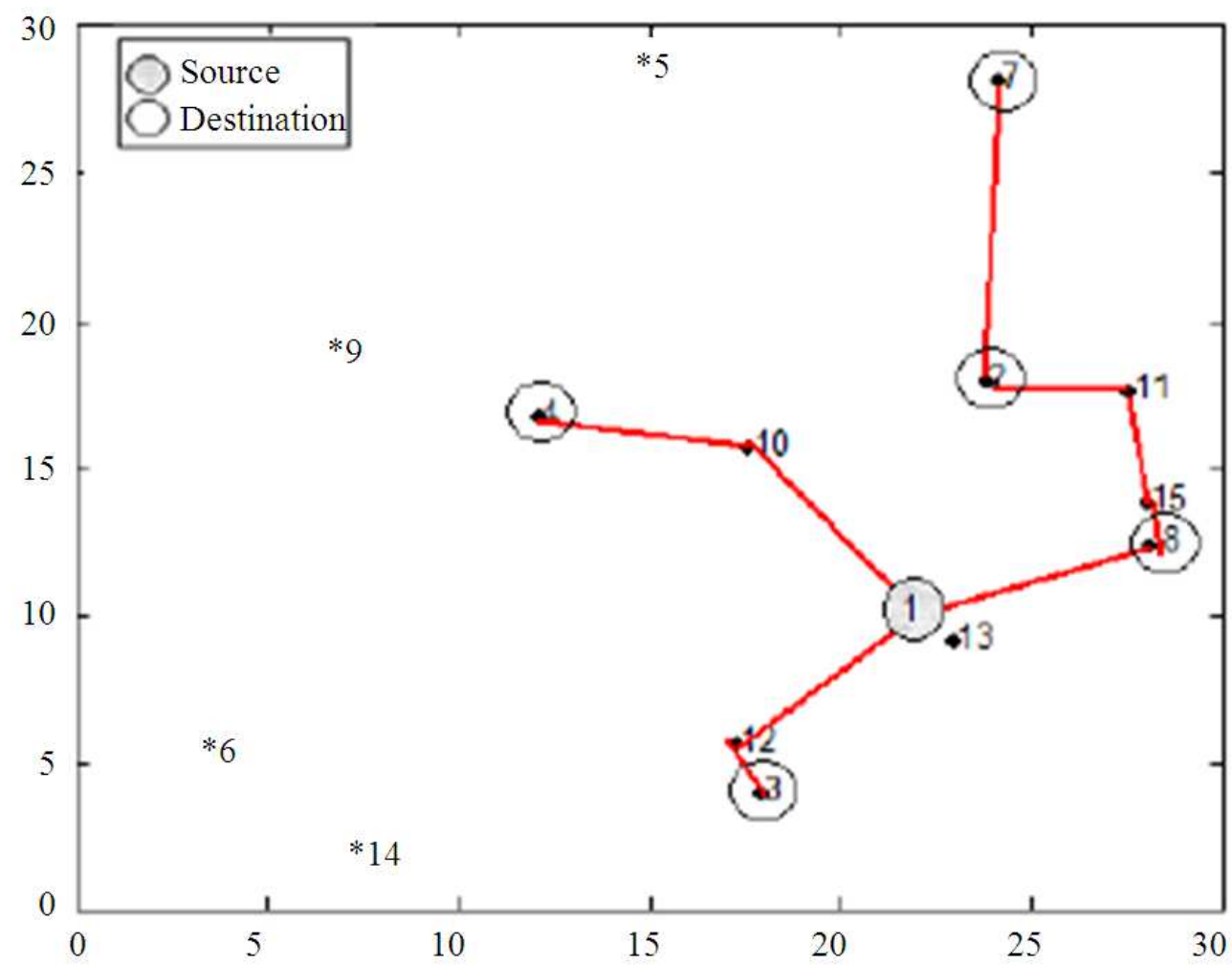

Fig. 6. The multicast tree for destinations $\{2,3,4,7,8\}$ with $\mu_{m}=0$ é. $4 \mathrm{~B}, \mathrm{P}_{\text {total }}=302.5019$

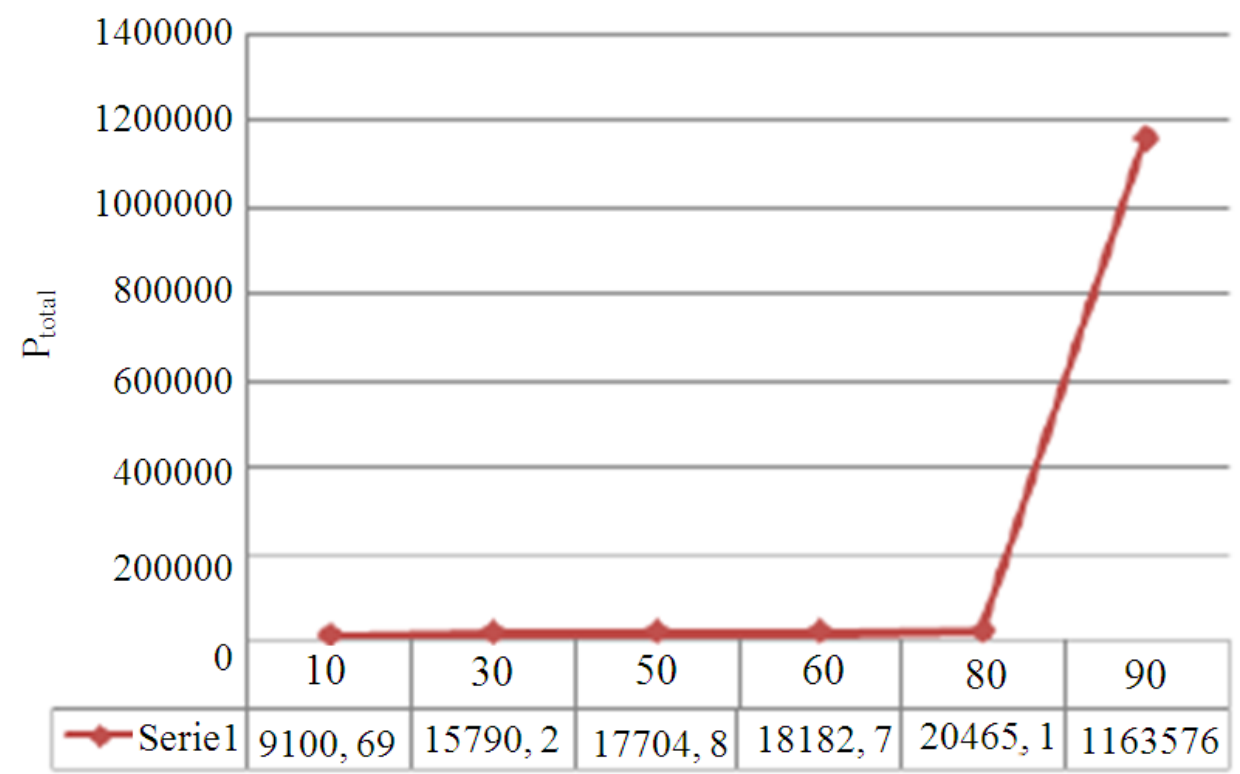

Fig. 7. the total energy for multicast sessions with different $\mathrm{M}$ size and $\lambda_{\mathrm{m}}=0.01 \mathrm{~B}$ 


\section{CONCLUSION}

In this study, we have presented a constraint formulation for the QoS routing topology control problem in wireless ad hoc networks. It is based on a formulation for the minimum energy multicast routing. The problem has been formulated in a form of integer linear programming problem with QoS parameters. We implemented the proposed ILP to construct a multicast tree that minimizes the total energy cost in a network and satisfies also the two constraints of QoS: Bandwidth and delay. We conducted several simulations to evaluate the performance of the proposed algorithm.

The proposed algorithm has proved his ability to construct multicast topologies respecting QoS constraints. Nevertheless, when applied to large networks, it induces time complexity and difficulty to find a connected topology which satisfies the input requests. Therefore, further research should be made to obtain solutions able to deal with large networks.

\section{REFERENCES}

Badis, H., I. Gawedzki and K. Al Agha, 2004. QoS routing in ad hoc networks using QOLSR with no need of explicit reservation. Proceedings of the 60th Vehicular Technology Conference, Sept. 26-29, IEEE Xplore Press, pp: 2654-2658. DOI: 10.1109/VETECF.2004.1400538

Bauer, J., D. Haugland and D. Yuan, 2008. Analysis and computational study of several integer programming formulations for minimum-energy multicasting in wireless ad hoc networks. Networks, 52: 57-68. DOI: $10.1002 /$ net.20222

Chen, H. and B. Sun, 2005. Multicast routing optimization algorithm with bandwidth and delay constraints based on GA. J. Commun. Comput., 2: 63-67.

Chen, S. and K. Nahrstedt, 1999. Distributed quality-of service routing in ad hoc networks. IEEE J. Selected Areas Commun, 17: 1488-1505. DOI: $10.1109 / 49.780354$

IBM ILOG CPLEX V12.1, 2009. User's Manual. IBM ILOG CPLEX V12.1

Das, A.K., R.J. Marks, M. El-Sharkawi, P. Arabshahi and A. Gray, 2003. Minimum power broadcast trees for wireless networks: Integer programming formulations. Proceedings of the 22nd Annual Joint Conference of the IEEE Computer and Communications Societies, Mar. 30-Apr. 3, IEEE Xplore Press, pp: 1001-1110. DOI: 10.1109/INFCOM.2003.1208937
Guo, S. and O. Yang, 2004. Minimum energy multicast routing for wireless ad-hoc networks with adaptive antennas. Proceedings of the 12th IEEE International Conference on Network Protocols, Oct. 5-8, IEEE Xplore Press, pp: 151-160. DOI: 10.1109/ICNP.2004.1348106

Huang, Z., C.C. Shen, C. Scrisathapornphat and C. Jaikaeo, 2002. Topology control for Ad Hoc networks with directional antennas. Proceedings of the IEEE 11th Conference on Computer Communications and Networks, Oct. 14-16, IEEE Xplore Press, , pp: 16-21. DOI: 10.1109/ICCCN.2002.1043039

Idrissi, A., 2012. How to minimize the energy consumption in mobile ad-hoc networks. Int. J. Artificial Intell. Applic., 3: 1-10. DOI: 10.5121/ijaia.2012.3201

Idrissi, A., C.M. Li, J.F. Myoupo, 2006. An algorithm for a constraint optimization problem in mobile adhoc networks. Proceeding of the 18th IEEE International Conference on Tools with Artificial Intelligence, Nov. 13-15, IEEE Xplore Press, Arlington, VA., pp: 555-562. DOI: 10.1109/ICTAI.2006.29

Jia, X., D. Li and D.Z. Du, 2004. QoS topology control in ad hoc wireless networks. Proceeding of the 23 Annual Joint Conference of the IEEE Computer and Communications Societies, Mar. 7-11, IEEE Xplore Press, pp: 1264-1272. DOI: 10.1109/INFCOM.2004.1357012

Kawadia, V. and P.R. Kumar, 2003. Power control and clustering in ad hoc networks. Proceedings of the 22th Annual Joint Conference of the IEEE Computer and Communications, Apr. 30-3, IEEE Xplore Press, pp: 459-469. DOI: 10.1109/INFCOM.2003.1208697

Li, L., J.Y. Halpern, P. Bahl, Y.M. Wang and R. Wattenhofer, 2001. Analysis of a cone-based distributed topology control algorithm for wireless multi-hop networks. Proceedings of the 20th Annual ACM Symposium on Principles of Distributed Computing, Aug. 26-28, Newport, RI, USA, pp: 264- 273. DOI: $10.1145 / 383962.384043$

Li, N., J.C. Hou and S. Lui, 2003. Design and analysis of an MST-based topology control algorithm. Proceedings of the 22nd Annual Joint Conference of the IEEE Computer and Communications Societies, Apr. 30-3, IEEE Xplore Press, pp: 1702-1712. DOI: 10.1109/INFCOM.2003.1209193 
Lloyd, E.L., R. Liu, M.V. Marathe, R. Ramanathan and S.S. Ravi, 2005. Algorithmic aspects of topology control problems for ad hoc networks. Mob. Netw. Applic., $\quad 10$ : 19-34. DOI: 10.1023/B:MONE.0000048543.95178.f5

Marsan, M.A., C.F. Chiasserini, A. Nucci, G. Carello and L.D. Giovanni, 2002. Optimizing the topology of Bluetooth wireless personal area networks. Proceedings of the IEEE 21st Annual Joint Conference of the IEEE Computer and Communications Societies, Jun. 23-27, IEEE Xplore Press, pp: 572-579. DOI: 10.1109/INFCOM.2002.1019302

Maruthamuthu, S. and S. Sankaralingam, 2011. Energy aware multiple constraints quality of service routing protocol with dynamic mobility prediction for mobile ad hoc networks. J. Comput. Sci., 7: 892901. DOI: $10.3844 /$ jcssp.2011.892.901

Pan, Y. and Y. Xiao, 2006. Ad hoc and sensor networks.

Ramanathan, R. and R. Rosales-Hain, 2000. Topology control of multihop wireless networks using transmit power adjustment. Proceedings of the 19th Annual Joint Conference of the IEEE Computer and Communications Societies, Mar. 26-30, IEEE Xplore Press, Tel Aviv, pp: 404-413. DOI: 10.1109/INFCOM.2000.832213

Sabari, A. and K. Duraiswamy, 2009. Multiple constraints for ant based multicast routing in mobile ad hoc networks. J. Comput. Sci., 5: 1020-1027. DOI:10.3844/jcssp.2009.1020.1027
Singh, S., M. Woo and C.S. Raghavendra, 1998. Poweraware routing in mobile ad hoc networks. Proceedings of the 4th Annual ACM/IEEE International Conference on Mobile Computing and Networking, Oct. 25-30, Dallas, TX, USA, pp: 181190. DOI: $10.1145 / 288235.288286$

Thenmozhi, D.S. and R. Lakshmipathi, 2010. Highly ensured QoS routing in mobile ad hoc networks based on multiple constraints. Int. J. Comput. Applic., 8: 23-29. DOI: 10.5120/1199-1702

Wan, P.J., G. Calinescu, X.Y. Li and O. Frieder, 2001. Minimum-energy broadcast routing in static ad hoc wireless networks. Proceedings of the 20th Annual Joint Conference of the IEEE Computer and Communications Societies, (CCS' 01), IEEE Xplore Press, Anchorage, AK, pp: 1162-1171. DOI: 10.1109/INFCOM.2001.916310

Wieselthier, J.E., G.D. Nguyen and A. Ephremides, 2000. On the construction of energy-efficient broadcast and multicast trees in wireless networks. Proceedings of the 19th Annual Joint Conference of the IEEE Computer and Communications Societies, (CCS' 00), IEEE Xplore Press, Tel Aviv, pp: 585594. DOI: 10.1109/INFCOM.2000.832232

Wu, H. and X. Jia, 2007. QoS multicast routing by using multiple paths/trees in wireless ad hoc networks. Ad Hoc Netw., 5: 600-612. DOI: 10.1016/j.adhoc.2006.04.001 